\title{
On Everyone's lips: Insects for food and feed
}

\author{
Thomas Klammsteiner ${ }^{1}$ \\ Department of Microbiology, University of Innsbruck \\ Technikerstraße 25d, 6020 Innsbruck, Austria \\ E-mail: thomas.klammsteiner@uibk.ac.at
}

\section{Andreas Walter}

Department of Biotechnology \& Food Engineering, MCI - The Entrepreneurial School Maximilianstraße 2, 6020 Innsbruck, Austria

E-mail: andreas.walteremci4me.at

\section{Heinrich Pan}

Spielraum Fablab

Franz-Fischer-Straße 12, 6020 Innsbruck, Austria

E-mail: h.pan@mailbox.org

\section{Magdalena Gassner}

Department of Microbiology, University of Innsbruck

Technikerstraße 25d, 6020 Innsbruck, Austria

E-mail: magdalena.gassnerestudent.uibk.ac.at

\section{Carina Desirée Heussler}

Department of Microbiology, University of Innsbruck

Technikerstraße 25d, 6020 Innsbruck, Austria

E-mail: carina.heussler@uibk.ac.at

\section{Markus Schermer}

Department of Sociology, University of Innsbruck

Universitätsstraße 15, 6020 Innsbruck, Austria

E-mail: markus.schermer@uibk.ac.at

\section{Heribert Insam}

Department of Microbiology, University of Innsbruck

Technikerstraße 25d, 6020 Innsbruck, Austria

E-mail: heribert.insam@uibk.ac.at 
Innovative businesses and a growing body of research have put their eyes on insect-based solutions to produce high-quality feed and food. One of the forerunner species in this trend is the Black Soldier Fly (BSF; Hermetia illucens) - a low maintenance tropical insect capable of efficiently converting organic wastes into insect biomass that mainly consists of protein, fat and chitin. However, the exploitation of insects in western countries is still in its infancy, but an improving legislation as well as scientific advancements support the growing insect industry. By offering hands-on workshops to schools and the public, we aimed at getting people in touch with the BSF to raise awareness about the re-valorisation of organic waste and sustainable feed production. Participants were invited to build a take-home BSF rearing system running on their daily occurring household bio-waste. Within these three-week home experiments, citizen scientists successfully collected data on the amount and composition of fed bio-waste, larval development and substrate maturation. Moreover, carrying out the same feeding experiment under different environmental circumstances provided insights in the adaptiveness of the larvae of this fly and will further help to improve rearing conditions.

5th Austrian Citizen Science Conference 2019 (ACSC2019)

26-28, June, 2019

Obergurgl, Austria 


\section{Introduction}

The call for novel sustainable protein and energy sources to supply aquaculture and traditional livestock farming is louder than ever, questioning the current global use of soy- and fishmeal as major nutrient sources for animal feed [1]. Over the past decade, many studies focussed on the suitability of insects for food and feed as ecologically reasonable alternative [2]. But at the same time, the EU-wide legal framework regulating the circulation of insectbased products is only slowly adapting [3].

Our citizen-science-based approach offered a novel way to involve the public in this topic and inspired an open-minded "out of the box" thinking on a scientific level. We developed a low-budget and easy to handle system to simulate the process of growing Black Soldier Fly (BSF; Fig. 1) larvae, which are commonly used for waste conversion and feed production [4]. In free open workshops we introduced people to the biology of this insect and had them supervise their own three-week feeding experiments at home. We hypothesized that home-rearing of BSF larvae with distinct compositions of organic waste, shaped by the dietary habits of citizen scientists, will allow for similar biomass

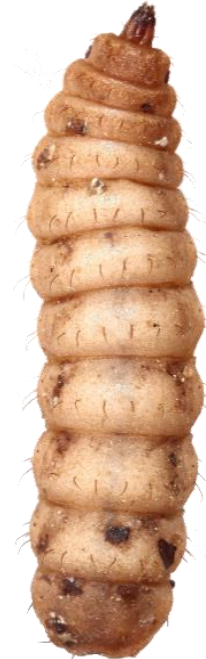

Fig. 1 - Hermetia illucens larva Photo: W. Dibiasi gain and development as observed under other artificial conditions.

\section{House for the critters - components and functioning}

The rearing system for the larvae consisted of two main parts - a house-like exterior lasercut from medium-density fibreboards and an inner plastic container taking up larvae and biowaste (Fig. 2). A laboratory journal for observations, 200 young BSF larvae, a pocket fine scale (capacity/grading: $200 \mathrm{~g} / 0.01 \mathrm{~g}$ ), and forceps were provided for each participant at the beginning of the experiment. The plastic container was equipped with a drainage hole connected to a detachable glass receptacle to avoid water accumulation from excessively humid bio-waste. A ramp with an approx. $40^{\circ}$ slope within the plastic container provided an escape route for migrating larvae (so called self-harvesting ability) once they reached the final instar and seeked for a sheltered hiding spot for pupation. The ramp guided the larvae through a tube leading out of the

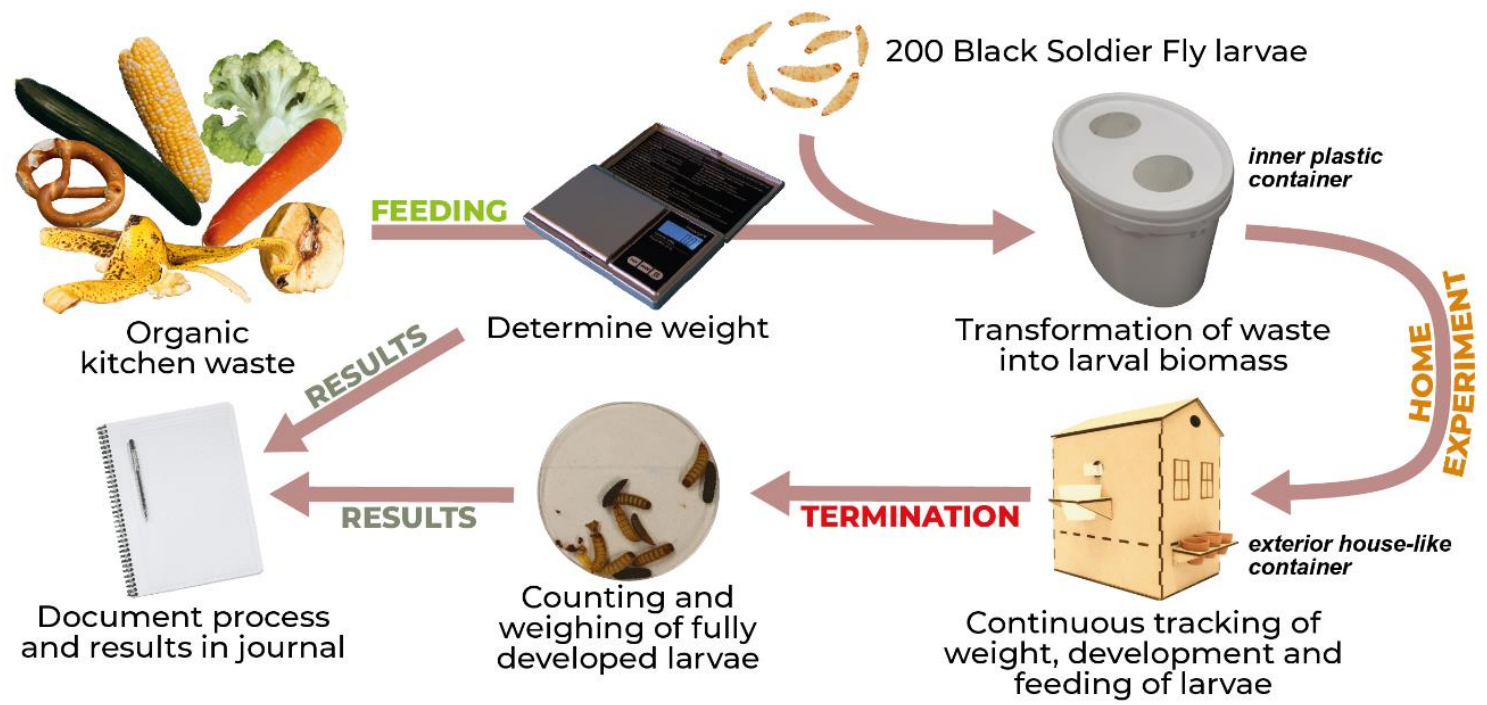

Fig. 2 - Workflow for the home experiment. 
house into an escape-proof collection cup mounted on the houses' façade. Feeding and sampling was scheduled for every second to third day and consisted of adding 30-40 g organic waste $\mathrm{d}^{-1}$ as feed and weighing five randomly collected larvae to determine biomass increase.

\section{Planning and implementation of workshops}

Workshops for schools and the public were both conceptualized in a similar fashion and scheduled for three to four hours depending on the size of the group. In schools, additional emphasis was put on group work and the integration of the project within the syllabus. Each workshop started with a survey on the attendees' attitude towards insects and products therefrom. This was followed by a 45 minutes lecture on the biology of the BSF and the context of its use in waste valorisation and feed production. The subsequent practical part consisted of the assembly of the rearing system and a trial feeding including instructions on how to collect data in a scientifically correct manner (Fig. 2). Each house was supervised by the responsible participant(s) (either a group of pupils in schools or a single citizen in households) for the duration of three weeks. To terminate the experiments, the houses were retrieved from their hosts and the surviving larvae and residual waste fractions were evaluated and appropriately stored for further analyses. Feedback on the workshops and rearing system was gathered in a final discussion with most of the participants.

\section{Results and outlook}

We reached more than 110 school kids from five different types of schools and 30 citizens scientists conducted the experiment at home. Workshops in schools included pupils ranging from 14-18 years old, while the participant's age in public workshops averaged at $37 \pm 15$ years. We laser-cut and built more than 40 rearing systems, whereas the sturdy material and design allowed for repeated dis- and reassembly of the same units. In total, over 10,000 single BSF larvae were counted. In $80 \%$ of the experiments (total $n=27$ ) at least 180 of the 200 larvae survived the three weeks and $31 \pm 29 \%$ already progressed to pupal stage at the end of the trial. The citizen scientists recorded on average a 15-fold biomass increase from initial larval weight (12 $\pm 1 \mathrm{mg}$ per larva) to the point of experiment termination $(190 \pm 50 \mathrm{mg}$ per larva) after $23 \pm 1.5$ days. The high survival rate as well as a fast and extensive biomass increase can be interpreted as success for the home trials and as a proof of concept for the rearing system. Similar feeding experiments carried out under artificial conditions found comparable larval weights, survival and developmental times $[5,6]$. Participants mostly carried out the experiment in their apartment (12 of 27) or on their balcony (9 of 27) and stated mean temperatures of $24 \pm 2$ and $17 \pm 1{ }^{\circ} \mathrm{C}$, respectively. These temperatures were below the $27{ }^{\circ} \mathrm{C}$ commonly used in artificial rearing [5]. Heat production during larval and microbial degradation as well as insulation provided by the waste could have supported larval activity. All materials needed to reproduce similar workshops including blueprints for laser-cutting the houses will be made available on our website (www.fromwastetofeed.wordpress.com)

Although the experimental scope only covered the growth and pupation of larvae without including the adult and egg stage, we are convinced that participants gained insight into strategies on how to tackle socio-ecological questions using insects. Not only the participants profited from this experience, but their data on larval development will also contribute to the optimization of small-scale rearing. Moreover, the collected larvae, pupae and waste residues will be analysed in 
a follow-up project to investigate the impact of feed patterns and human co-habitation on the microbiome in and on the larvae.

\section{Acknowledgements}

We thank Univ.-Prof. Susanne Kapelari, Dr. Pamela Vrabl, Wolfgang Dibiasi and all the participating teachers, pupils and citizen scientists for their valuable input and suggestions.

This Top Citizen Science project was funded by the Austrian Science Fund (FWF; project number: TCS48).

\section{References}

[1] Food and Agriculture Organization of the United Nations (FAO), The state of world fisheries and aquaculture 2018 - Meeting the sustainable development goals, FAO, Rome 2018.

[2] S. Govorushko, Global status of insects as food and feed source: A review, Trends Food Sci Technol 91 (2019) 436-445.

[3] M.D. Finke, S. Rojo, N. Roos, A. van Huis, A.L. Yen, The European Food Safety Authority scientific opinion on a risk profile related to production and consumption of insects as food and feed, J Insect Food Feed 01 (2015) 245-247.

[4] B. Pastor, Y. Velasquez, P. Gobbi, S. Rojo, Conversion of organic wastes into fly larval biomass: bottlenecks and challenges, J Insect Food Feed 01 (2015) 179-193.

[5] J.K. Tomberlin, D.C. Sheppard, J.A. Joyce, Selected life-history traits of black soldier flies (Diptera: Stratiomyidae) reared on three artificial diets, Ann Entomol Soc Am 95 (2002) 379-386.

[6] T.T.X. Nguyen, J.K. Tomberlin, S. Vanlaerhoven, Ability of black soldier fly (Diptera: Stratiomyidae) larvae to recycle food waste, Environ Entomol 44 (2015) 406-410. 